\title{
Terahertz Harmonic Operation of Microwave Fresnel Zone Plate Lens and Antenna: Frequency Filtering and Space Resolution Properties
}

\author{
Hristo D. Hristov \\ Departamento de Electrónica Engineering, Universidad Técnica Federico Santa María, Valparaíso, Chile \\ Correspondence should be addressed to Hristo D. Hristov, hristo.hristov@usm.cl \\ Received 1 July 2011; Revised 9 September 2011; Accepted 11 September 2011 \\ Academic Editor: Krishnasamy Selvan \\ Copyright (C) 2011 Hristo D. Hristov. This is an open access article distributed under the Creative Commons Attribution License, \\ which permits unrestricted use, distribution, and reproduction in any medium, provided the original work is properly cited.

\begin{abstract}
This paper examines the binary Fresnel zone plate (FZP) lens frequency-harmonic and space-resolution focusing, and its application as a FZP lens antenna. A microwave FZP lens antenna (FZPA) radiates both at design $(90 \mathrm{GHz})$ and terahertz (THz) odd harmonic frequencies. Frequency and space domain antenna operation are studied analytically by use of the vector diffraction integral applied to a realistic printed FZPA. It is found that all harmonic gain peaks are roughly identical in form, bandwidth, and top values. At each harmonic frequency, the FZPA has a beamwidth that closely follows the Rayleigh resolution criterion. If the lens/antenna resolution is of prime importance and the small aperture efficiency is a secondary problem the microwave-design FZP lens antenna can be of great use at much higher terahertz frequencies. Important feature of the microwave FZP lens is its broaderzone construction compared to the equal in resolution terahertz-design FZP lens. Thus, unique and expensive microtechnology for the microwave FZP lens fabrication is not required. High-order harmonic operation of the FZP lens or lens antenna could find space resolution and frequency filtering applications in the terahertz and optical metrology, imaging tomography, short-range communications, spectral analysis, synchrotron facilities, and so on.
\end{abstract}

\section{Introduction}

During the last two decades, a rapid exploration of terahertz waves is taking place [1-3]. Fresnel zone plate (FZP) lenses, binary and phase correcting, have already turned into essential focusing and imaging elements in the microwave and terahertz electronic systems [4-13]. At terahertz frequencies, the multilevel phase-correcting dielectric FZP lens shows better efficiency compared to the ordinary dielectric lens, and is easier and cheaper to fabricate because of its planar configuration [4-6].

If the high lens resolution and small thickness are of prime importance, the binary FZP lens consisting of freestanding or printed skinny metal rings would be preferable. Such lenses have found a lot of applications in the areas of 3D imaging tomography, electron microscopy, sensing and security systems, and synchrotron radiation facilities for beam focusing [11, 14-19]. For these purposes, a special lithography process has been developed for fabrication of high aspect ratio gold structures on silicon nitride membranes [19].
Because the classical FZP lens is acting as a diffraction grating of annular elements, it possesses very intriguing spectral periodicity $[5,9,11,13,16]$.

Main objective of this paper is to clarify the harmonic action of binary FZP lens and antenna, and to examine them numerically in frequency and space domains with for grounding hypothetically their future employment as multiband focusing and filtering devices.

Section 2 provides shortly the FZP lens design and resolution basics and makes physical clarification of the harmonic-mode FZP focusing mechanism.

Section 3 illustrates the microwave FZP antenna radiation geometry used in the vector Kirchoff's diffraction theory for the axially symmetric FZP lens antenna analysis and optimization. The particular $90 \mathrm{GHz}$ printed FZP lens antenna design described in this section operates in a periodic way at much higher frequencies in accordance with the odd harmonic sequence $f_{1}, 3 f_{1}, 5 f_{1}$, and so forth, where $f_{1}=90 \mathrm{GHz}$ is the antenna design (first order) microwave frequency. For the first time, the FZP antenna space-domain 
operation is studied for finding antenna vector radiation field and resolution characteristics at the design (microwave) and higher (terahertz) harmonic frequencies.

Section 4 describes FZP lens antenna designed for the terahertz frequency of $1530 \mathrm{GHz}$. Its radiation, structural, and technological properties are observed and contrasted to those of the same-size $90 \mathrm{GHz}$ microwave FZP lens antenna operating at its 15 th terahertz harmonic frequency of $1530 \mathrm{GHz}$ as well.

The paper is completed by a general conclusion, acknowledgement, and list of references.

\section{Harmonic-Mode Operation of FZP Lens Illuminated by Plane Wave}

2.1. FZP Design. In general, a FZP lens centered in the origin of Cartesian coordinate system is transforming an axially incident spherical wave front of radius $z=-F_{s}$ into multiple spherical waves focused on the lens axis $\mathrm{z}$ at multiple foci with focal distances (lengths) $z=+F_{n}$, where $n=1,3,5, \ldots$ are odd numbers. The unusual multiple focusing action of the FZP lens is due to its diffractive nature, and this is its major distinction from the ordinary refractive lens. The first or primary focus for which $n=1$ has a focal distance $F_{1}$ collects the largest portion of the focused field energy in the forward semispace $z>0$. This allows the others or so-called secondary foci at $z=F_{3}=F_{1} / 3, z=F_{5}=F_{1} / 5$, and so on to be neglected.

Figure 1 illustrates a binary-amplitude FZP lens of diameter $D$ illuminated along the axis $z$ by a plane wave that corresponds to $F_{s}=-\infty$. With the secondary foci disregarded, the lens will have a single focus at a focal distance $F=F_{1}$. Such a lens is operating in the antenna receive mode. The FZP lens drawn in Figure 1 has odd zones closed (tinted in black).

The normally illuminated by a plane wave planar FZP conmprises concentric half-wave zones with zone radii $b(m)$ determined by

$$
b(m)=\sqrt{m \lambda F+\left(\frac{m \lambda}{2}\right)^{2}},
$$

where $\lambda$ is the design wavelength, $m=1,2,3, \ldots, M$ is the current zone number, and $M$ is the ending zone number. valid:

In optics the following approximation of (1) is usually

$$
b(m)=\sqrt{m \lambda F}=b(1) \sqrt{m},
$$

where the term $(m \lambda / 2)^{2}$ is neglected because the ratio $m \lambda F \gg(m \lambda / 2)^{2}$ takes place, and $b(1)=\sqrt{\lambda F}$ is the first zone radius.

2.2. FZP Lens Resolution. By definition, the so-called Rayleigh resolution criterion for the resolving power of a circular aperture of diameter $D$ is typically expressed by the resolving angle $\delta_{0}$, in radians [20-22]

$$
\delta_{0}=\chi \frac{\lambda}{D}
$$

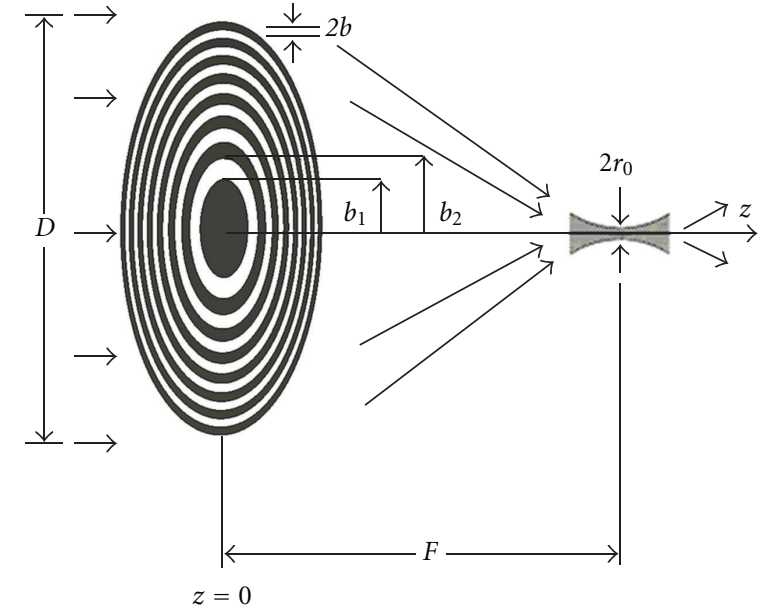

FIGURE 1: Plane-wave illuminated binary negative Fresnel zone plate lens.

where $\chi=1.22$ is a constant that is valid for every open circular aperture illuminated normally by a plane wave. This can be the aperture of a circular horn, parabolic reflector, ordinary refractive, or phase-correcting FZP lens.

From (3), is evident that if the diameter $D$ is maintained constant, the resolving angle is proportional to the wavelength $\lambda$ and $\chi$. It is said that the smaller angle $\delta_{0}$ the bigger lens resolution, and vise versa.

In the case of a binary FZP, however, the constant value 1.22 of $\chi$ is valid only if the total number of zones $M$ is bigger than about 200. For smaller $M$, say $M<50, \chi$ differs from 1.22 and varies with $M$, or $\chi=\chi(M)[23,24]$. More exactly, $\chi(M)>1.22$ for the positive FZP lenses (odd zones open), and $\chi(M)<1.22$ for the negative FZP lenses (odd zones closed). In Table 1 , several values of $\chi(M)$ for positive (pFZP) and negative (nFZP) lenses are listed $[22,23]$.

From Table 1, it is concluded that for $M \leq 50$ and the same normalized aperture diameter $D / \lambda$, the negative FZP has a smaller resolving angle $\delta_{0}$ (or bigger resolution) than the positive FZP and ordinary refractive lens by $15 \%$ and 9.5\%, for $M=10,8 \%$ and $5.5 \%$ for $M=20,5 \%$ and $3.5 \%$, for $M=30,3.5 \%$ and $2.5 \%$, for $M=40$, and $2.7 \%$ and $2 \%$, for $M=50$, respectively.

The resolving angle $\delta_{0}$ is directly related to the first null radius $r_{0}$ of the FZP Airy diffraction pattern at the focal plane. The radius $r_{0}$ measures the space (distance) lens resolution and is opposite to angle $\delta_{0}$ in the corresponding rignt triangle, so that $\tan \delta_{0}=r_{0} / F$. For a high resolution, the angle $\delta_{0}$ is small enough, or $\delta_{0} \approx r_{0} / F$. Thus, taking into account (3) the resolving lens $r_{0}$ can be expressed as

$$
r_{0}=\chi(M) \frac{\lambda F}{D}
$$

The ending zone width $\Delta b$ is related to the resolving lens radius as follows $[11,23,24]$ :

$$
r_{0}=\chi(M) \Delta b
$$


TABLE 1: Values of $\chi(M)$ for $M \leq 50$.

\begin{tabular}{|c|c|c|c|c|c|c|}
\hline \multicolumn{2}{|c|}{$M$} & 10 & 20 & 30 & 40 & 50 \\
\hline \multirow{2}{*}{$\chi(M)$} & pFZP & 1.275 & 1.245 & 1.235 & 1.230 & 1.227 \\
\hline & $\mathrm{nFZP}$ & 1.125 & 1.165 & 1.185 & 1.195 & 1.200 \\
\hline
\end{tabular}

or vice versa

$$
\Delta b=\frac{r_{0}}{\chi(M)} .
$$

2.3. Multifrequency Focusing of FZP Lens: Physical Justification. Next, we clarify the multiple frequency-domain behavior of the binary FZP lens illuminated by a plane wave. This phenomenon is analogious to the axial multiple focusing of the same lens. Both phenomena are linked by (1) and can be easier explained by its approximation (2). From (2), it follows that for a given FZP diameter $D=2 b(M)$, the change of the focal distance $F$ for $\lambda=\lambda_{1}=$ const., or the wavelength $\lambda$ for $F=$ const. will raise a multifocal or multifrequency effect, respectively, if the total number of zones $M$ somehow changes. Here $\lambda_{1}$ is the design wavelength. This statement might be confusing having in mind that for a realistic FZP design, $D$ and $M$ are both constant quantities.

Next, it is clarified that the multifrequency phenomenon taking as an example a binary FZP lens of four Fresnel (or half-wave) zones designed for a focal length $F$ at a frequency $f_{1}$ (or wavelength $\lambda_{1}$ ). Consider first this FZP lens illuminated by a plane wave of frequency equal to the third harmonic frequency $f_{3}=3 f_{1}$, corresponding to the freespace wavelength $\lambda_{3}=\lambda_{1} / 3$. The radius (2) could be rewritten for the FZP diameter as follows:

$$
D\left(M_{3}\right)=2 \sqrt{M_{3} F \lambda_{3}} .
$$

The maintenance of same aperture diameter $D$ and focal length $F$ for the three times smaller wavelength $\lambda_{3}$ requires three times bigger number of zones, or $M_{3}=3 \mathrm{M}$. This means that in each real zone corresponding to the design wavelength $\lambda_{1}$ three virtual $\lambda_{3}$-subzones should be enclosed: two positive (in white) and one negative (in grey). Thus, the virtual subzone radii can be calculated by the equation $b\left(m_{3}\right)=\sqrt{m_{3} \lambda_{3} F}=\sqrt{3 m \lambda_{3} F}$, where $m_{3}$ is the current subzone number. The above suggestion is illustrated by Figure 2(a), where in each open realistic zone, three virtual subzones are included. The fields produced by the 2nd subzone and 3rd subzone will be canceled leaving only the 1 st subzone to contribute at the focus.

Similarly, for an illuminating plane wave with a frequency $f_{5}=5 f_{1}$, or wavelength $\lambda_{5}=\lambda_{1} / 5$, each real open zone should contain five virtual subzones: three positive and two negative, with radii found by $b\left(m_{5}\right)=\sqrt{5 m \lambda_{5} F}$. The two positive (3rd and 5th) and two negative (2nd and 4th) subzones will cancel at the primary focus leaving again only one, the 1st positive half-wave subzone to be productive. The above considerations are valid for all higher harmonic frequencies.

The field intensities provided at the primary focal point by all real open zones corresponding to $f_{1}$ and by all virtual
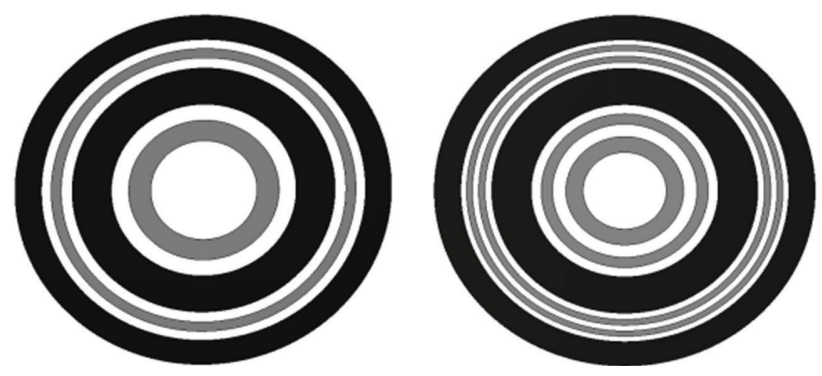

FIGURE 2: Harmonic-action FZP lens at (a) 3rd harmonic frequency $f_{3}$ and (b) 5 th harmonic frequency $f_{5}$.

constructive subzones related to any odd harmonic frequency are expected to be roughly equal. This is because the total constructive areas, the real $A_{1}$ and the virtual $A_{3}, A_{5}, \ldots$, normalized by corresponding squared wavelengths $\lambda_{1}^{2}, \lambda_{3}^{2}, \lambda_{5}^{2}, \ldots$, respectively, are about the same (or $A_{1} / \lambda_{1}^{2} \approx A_{3} / \lambda_{3}^{2} \approx A_{5} / \lambda_{3}^{2} \approx \cdots \approx$ const.). In other words, the focusing gain $G_{f}$ of all constructive zones, actually related to the design frequency, and virtually related to any harmonic frequency, could be considered equal.

Taking into account the basic lens/antenna relation between the focusing gain and radiating aperture $G_{f}=$ $4 \pi A \eta / \lambda^{2}$, the radiation aperture efficiency for each frequency is expressed as follows: $\eta_{1}=G_{f} \lambda_{1}^{2} / 4 \pi A_{1}, \eta_{3}=G_{f} \lambda_{3}^{2} / 4 \pi A_{1}$, $\eta_{5}=G_{f} \lambda_{5}^{2} / 4 \pi A_{1}, \ldots$, and so forth. It is seen that the efficiency values $\eta_{1}, \eta_{3}, \eta_{3}, \ldots$ are directly proportional to the respective ratios $\lambda_{1}^{2} / A_{1}, \lambda_{3}^{2} / A_{1}, \lambda_{5}^{2} / A_{1}, \ldots$. Because the physical aperture area of the lens $A_{1}$ and the focusing gain $G_{f}$ are considered constant quantities, the FZP aperture efficiencies for the $3 \mathrm{rd}$, 5th, and so forth harmonics are diminishing rapidly compared to the efficiency for the design frequency, or $\eta_{3}=$ $\eta_{1}(1 / 3)^{2}, \eta_{5}=\eta_{1}(1 / 5)^{2}$, and so on.

\section{Microwave FZP Lens Antenna Operation at Terahertz Harmonic Frequencies}

3.1. Radiation Characteristics Computed by Use of Vector Kirchhoff's Diffraction Theory. Consider the geometry of binary FZP lens antenna consisting of feed-horn and positive binary FZP of concentric metal rings (in grey) obstructing the even zones (Figure 3 ). The feed phase center coincides with the lens focal point $P_{1}(0,0,-F)$. In antenna transmit mode, the spherical wave that originated from $P_{1}$ illuminates the FZP lens which odd open Fresnel zones radiate constructively along the lens axis $(\theta=0)$.

The antenna radiation characteristics have been computed by use of the vector Kirchhoff's diffraction integral solved explicitly for the axially symmetric binary FZP lens antenna $[5,8,9]$.

The feed-horn field is approximated theoretically by the broadly used aperture directive gain function $G_{f}(\psi, n)=$ $G_{f}(0, n) \cos ^{n} \psi$ for $0 \leq \psi \leq \pi / 2$, where $G_{f}(0, n)=2(n+1)$, and $G_{f}(\psi, n)=0$ for $\pi / 2<\psi \leq \pi$. The lens aperture field is 


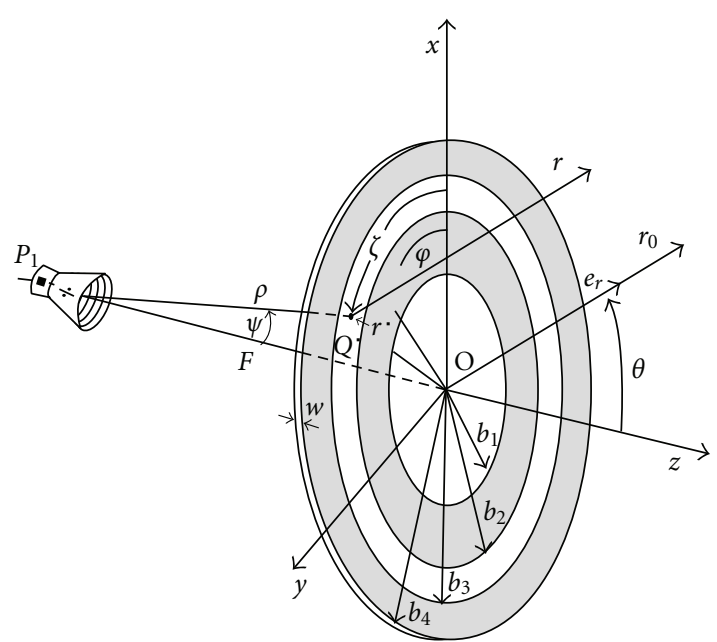

Figure 3: Radiation geometry of FZP lens antenna.

tapered to the edge level equal to $-10 \mathrm{~dB}$, or $10 \mathrm{~dB}$ down to the center field magnitude, $n=8.32$ and $G_{f}(0)=12.7 \mathrm{dBi}$.

The microwave FZP lens antenna design studied in this paper is marked by the acronym FZPA-M-10, which stands for FZP Antenna designed at the Microwave frequency $f_{1}=$ $90 \mathrm{GHz}$ and by having a FZP lens with 10 Fresnel zones in total (Figure 4). The lens has a diameter $D=100 \mathrm{~mm}$ and focal length $F=66.7 \mathrm{~mm}$, so the lens aspect ratio is $F / D=$ 0.67 .

The FZP lens is made as a printed construction with metal rings of thickness $t$ printed on a dielectric substrate of width $w$ and a complex permittivity $\tilde{\mathcal{E}}_{r}(f)=\varepsilon_{r}[1-$ $j \tan \delta(f)$ ], which is a function of frequency $f$. In the frequency band $\Delta f=f_{\max }-f_{\min }$, the loss tangent $\tan \delta$ of typical microwave dielectrics is roughly increased as a linear function of $f$ in the range $\Delta \tan \delta=\tan \delta_{\max }-\tan \delta_{\min }$ and can be approximated by the next simple equation:

$$
\tan \delta(f) \approx \tan \delta_{\min }+\mathrm{k}_{\Delta}\left(f-f_{\min }\right),
$$

where $\tan \delta_{\min }$ corresponds to $f_{\min }$, and $k_{\Delta}=\Delta \tan \delta / \Delta f$ is a constant specific for a given dielectric substrate. Equation (7) is an author's approximation for dielectrics with a linear conduct of the loss tangent versus frequency and is based on published measurements for some dielectrics in the millimeter-submillimer frequency bands [6]. For instance, in the $90 \div 1500 \mathrm{GHz}$ frequency band (8) matches well the loss tangent behavior of Teflon, chosen here as a FZP substrate dielectric, if $k_{\Delta}=0.0104$. The Teflon real permittivity $\varepsilon_{r}$ is approximately equal to 2.05 in the whole band. According to (8), for the working FZP frequencies 90, 270, and $1350 \mathrm{GHz}$, for instance, $\tan \delta$ has values $5 \cdot 10^{-4}, 6.8 \cdot 10^{-4}$, and 17.9 . $10^{-4}$, respectively.

In the studied FZP lens structure, the Teflon substrate plate is $1 \mathrm{~mm}$ thick, while the metal rings have a width of $0.1 \mathrm{~mm}$ and are supposed infinitely conductive.

The outmost (10th) zone is obstructed by a metal ring of width $\Delta b_{M}=2.83 \mathrm{~mm}$, which is slightly less than the wavelength corresponding to the design frequency of $90 \mathrm{GHz}$ but is about 10-15 times bigger than the wavelengths related to

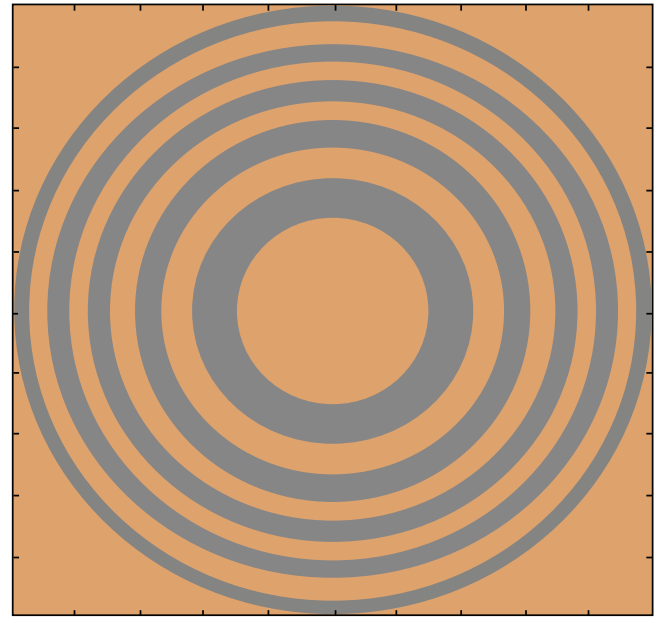

FIGURE 4: Binary FZP lens of FZPA-M-10 designed at microwave frequency of $90 \mathrm{GHz}$ with all even zones covered by thin metal (in grey) printed on dielectric substrate (in light brown).

highest harmonic frequencies in the band for which the FZPM-10 is studied here.

3.2. Frequency-Domain Performance of FZP Antenna. Frequency analysis of the FZP lens antenna is based on the variation of far-field directive gain versus frequency in the normal direction $f=90$ along the $z$-axis (or at $\theta=0 \mathrm{deg}$ ). The main frequency characteristics of interest are the antenna directive peak gain $\left(G_{p}, \mathrm{dBi}\right)$ and the $3-\mathrm{dB}$ gain bandwidth $(\mathrm{B})$ around the design and harmonic frequencies.

The FZPA-M-10 has been examined numerically in the frequency band 50-1550 GHz. Figure 5 illustrates the characteristic multiband comblike FZP focusing/radiation behavior with gain peaks at the microwave frequency of $90 \mathrm{GHz}$ and its odd terahertz harmonics of $270,450,630, \ldots, 1350$, and $1530 \mathrm{GHz}$.

The multiband FZP antenna is considered as a multitude of individual harmonic-frequency antennas radiating all or each at a time. The analysis is made on the assumption that each individual harmonic antenna is fed by a feed located at the same focal point $P_{1}$ and radiating at the respective odd harmonic. These imaginary feeds are supposed to have the same shape radiation patterns and directive gains. In fact, such an enclosure of several different sizes of feeds (feed horns, for example) in the small focal area is a very challenging construction.

Practically, the harmonic-frequency feeds could be also located off axis on a circular arc, which is the scan curve of the FZP lens antenna [20]. Another pragmatic feed might be a single frequency independent feed like the Archimedean or equiangular spiral antenna having a radiation pattern and input impedance match roughly preserved for a number of microwave/THz harmonic frequencies. And finally, same configuration narrowband feedantennas, designed for the basic and harmonic frequencies, might be positioned each at a time in the FZP focal area by a simple electromechanical 


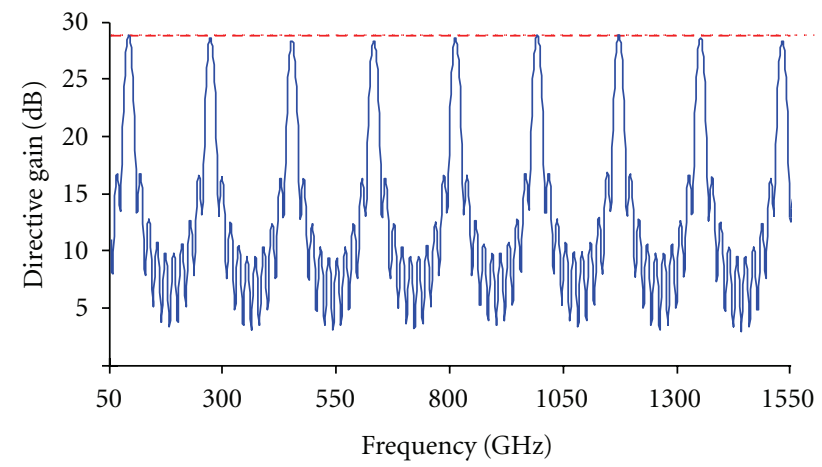

FIGURE 5: Frequency-domain harmonic action of binary FZP antenna: directive gain versus frequency.

TABLE 2: Frequency-domain radiation parameters of FZP lens antennas: microwave (M) and terahertz (T) designs.

\begin{tabular}{|c|c|c|c|c|c|}
\hline $\begin{array}{l}\text { FZP antenna } \\
F / D=0.67\end{array}$ & $\begin{array}{c}f \\
(\mathrm{GHz})\end{array}$ & $\begin{array}{c}G_{p} \\
(\mathrm{dBi})\end{array}$ & $(\mathrm{GHz})$ & $(\%)$ & $\begin{array}{c}e \\
(\%)\end{array}$ \\
\hline FZPA-M-10 & 90 & 28.9 & \multirow{3}{*}{$\sim 16.5$} & 18.4 & 9.63 \\
\hline$\left(f_{1}=90 \mathrm{GHz}\right.$ & 270 & 28.6 & & 6.1 & 1.07 \\
\hline$D=100 \mathrm{~mm})$ & 1350 & 28.5 & & 1.22 & 0.05 \\
\hline FZPA-T-150 & & & & & \\
\hline $\begin{array}{l}\left(f_{1}=1350 \mathrm{GHz}\right. \\
D=100 \mathrm{~mm})\end{array}$ & 1350 & 52 & 16.3 & 1.20 & 7.92 \\
\hline
\end{tabular}

device. Thus, every feedantenna will illuminate the FZP lens each at a time, or consecutively.

The essential frequency-domain parameters of the antenna FZPA-M-10 are listed in Table 2 for the design frequency $f_{1}=90 \mathrm{GHz}$ and for the two harmonic frequencies $f_{3}=270 \mathrm{GHz}$ and $f_{15}=1350 \mathrm{GHz}$. It is seen that the peak gain $G_{p}(\mathrm{~dB})$ has a slight fluctuation with the frequency due to the multiray transmission and absorption through the dielectric substrate, and the absolute bandwidth $B(\mathrm{GHz})$ is kept almost constant or $16.5 \mathrm{GHz}$, respectively. The normalized (relative) bandwidth $B(\%)$ and aperture efficiency $e$ quickly go down with the frequency rise.

3.3. Space-Domain or Radiation Pattern Study. Here by a space-domain antenna study, the FZP lens radiation pattern analysis at the design and harmonic frequencies is understood. Figure 6 illustrates the radiation characteristics of microwave antenna FZPA-M-10, namely: (a) copolar radiation pattern and (b) cross-polar radiation pattern in the small angular interval $\theta=0 \div 3 \mathrm{deg}$ at the diagonal cut plane $\varphi=45^{\circ}$, for the same three harmonic frequencies chosen in the previous section: 90, 270, and $1350 \mathrm{GHz}$. In addition to the peak gain value of about $29 \mathrm{dBi}$, other basic pattern parameters can be found: main lobe beamwidth $B W$ (deg) read at a level $3 \mathrm{~dB}$ down to the peak gain value, first side lobe level $L_{s}(\mathrm{~dB})$, and maximum cross-polarization $L_{\mathrm{cr}}$ measured within the main lobe beamwidth.

The half-power (or $-3 \mathrm{~dB}$ ) antenna beamwidth $B W$ is approximately equal to the lens aperture resolving angle $\delta_{0}$ [22], or

$$
B W \approx \delta_{0} .
$$

For the FZP design wavelength $\lambda_{1}$ and for the harmonic wavelengths $\lambda_{3}, \lambda_{5}, \ldots$, and so on, (3) for the resolving angle can be rewritten as follows:

$$
\delta_{0 i}=\chi(M) \frac{\lambda_{i}}{D}
$$

where $i=1,3,5, \ldots$ is an odd integer number.

Thus, for a known design FZP resolving angle $\delta_{01}$, the ith resolving angle $\delta_{0 i}$ is in a reverse proportion to $\delta_{01}$, or

$$
\delta_{0 i}=\frac{\delta_{01}}{h_{i}}
$$

where $h_{i}=f_{i} / f_{1}$ is the harmonic scale ratio.

Similarly, for the $i$ th harmonic resolving distance $r_{0 i}$, (4) and (6) can be rewritten, respectively, as follows:

$$
\begin{aligned}
& r_{0 i}=\chi(M) \frac{\lambda_{l} F}{D}, \\
& r_{0 i}=\chi(M) \frac{\Delta b}{i} .
\end{aligned}
$$

Table 3 summarizes the radiation (space) pattern characteristics of microwave antenna FZPA-M-10 at the design frequency of $90 \mathrm{GHz}$, and at its 3rd and 15th harmonics, found from Figures 6(a), and 6(b). From Table 3 several essential features of the FZP antenna space-domain behavior are discovered.

(i) Theoretical beamwidth matches very well the Rayleigh resolution criterion for all working frequencies. For example, at $f=f_{1}=90 \mathrm{GHz}$ the FZP lens 


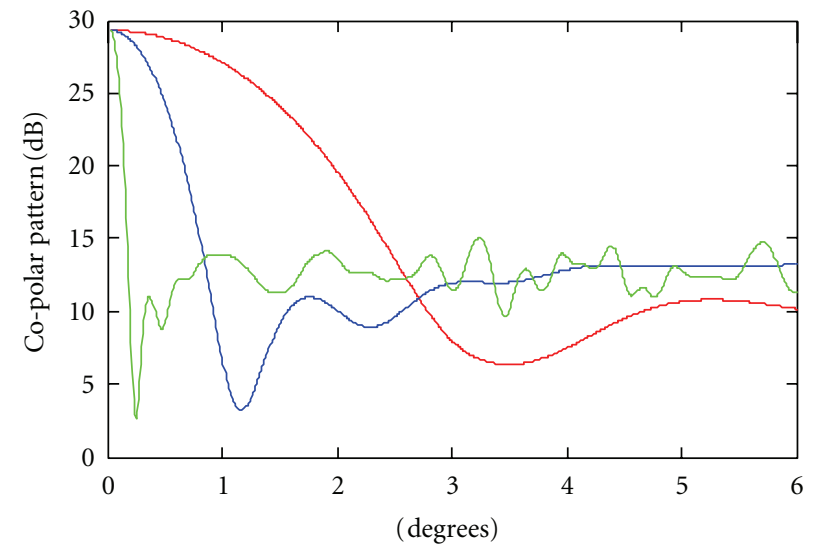

(a)

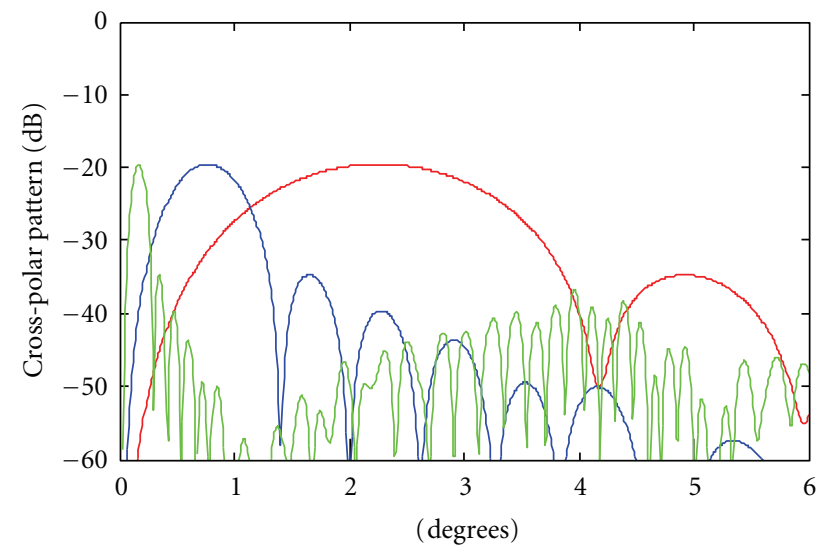

(b)

Figure 6: Radiation characteristics of FZPA-M-10 versus angle $\theta$ : (a) copolar radiation patterns, (b) cross-polar radiation patterns. Red, blue, and green lines correspond to 90,270 , and $1350 \mathrm{GHz}$, respectively.

antenna has a main lobe beamwidth $2.4 \mathrm{deg}$ that is roughly equal to the lens resolving angle $\delta_{01}=$ $2.43 \mathrm{deg}$. At the $3 \mathrm{rd}$ harmonic $(270 \mathrm{GHz})$ and 15 th harmonic $(1350 \mathrm{GHz})$ the beamwidth is 3 and 15 times much narrow: $B W_{3}=0.78 \mathrm{deg}$ and $B W_{15}=$ $0.15 \mathrm{deg}$. The same harmonic ratio $1: 3: 15$ is valid for the corresponding values of resolving angle $\delta_{0}$ and resolving distance $r_{0}$.

(ii) At all microwave and terahertz harmonics, the studied FZP lens antenna preserves roughly constant the peak gain values $\left(G_{p}=29 \mathrm{dBi}\right)$, first sidelobe level $\left(L_{s}=11 \mathrm{~dB}\right)$, and cross-polar level within the mainlobe beamwidth $\left(L_{\mathrm{cr}}=-25 \mathrm{~dB}\right)$.

The quick drop of the FZP lens/antenna focusing/ radiation efficiency with the rise of harmonic frequency deserves more discussions. The aperture antenna radiation efficiency less than $40-50 \%$ is not satisfactory, and less than $10-20 \%$ is considered small. In principle, the binary FZP lens/antenna belongs to the latter efficiency category because of noneffective amplitude and phase aperture utilization. By exploiting a proper phase correction of the aperture field like in the grooved dielectric or multidielectric FZP lenses, for instance, the corresponding FZP lens antenna easy reaches at its design (first-order) frequency the radiation efficiency of the classical aperture antennas (horn, parabolic-reflector, etc.), though in a rather smaller frequency band.

The low efficiency might be considered as a price for the unique features of the binary FZP lens or antenna: precise harmonic filtering and resolution characteristics that the usual directive-aperture antennas do not possess. Especially valuable is the big potential resolution at the much higher harmonic frequencies as it is discussed above. Similar "give and take" is met in many natural phenomena, and also in many other radioelectronic devices. Very appropriate example with a similar to the FZP multifrequency action is the harmonic-frequency multiplier. In particular, a big powerefficiency loss is produced in the process of harmonicfrequency multiplication of microwave frequencies to much higher terahertz frequencies. The output of the solid-state terahertz generators based on the frequency multiplication drops with harmonic frequency increase as about $(1 / f)^{3}[3]$. As is found here, the FZP lens/antenna radiation efficiency goes down like $(1 / f)^{2}$.

It is clear that at $\mathrm{THz}$ harmonic frequencies, the microwave FZP lens/antenna aperture is not utilized efficiently for a focusing or radiation. Such low aperture radiation efficiency, however, is also typical for all planar frequencyindependent antennas like the Archimedean and equiangular. For example, the Archimedean spiral antenna, which can radiate in a frequency band greater than $20: 1$ also shows very unproductive use of its radiation structure (aperture). At each frequency $f$ (or wavelength $\lambda$ ) only a narrow annular zone in the whole circular aperture radiates actively. This radiation zone has a radius of $\lambda / 2 \pi$ and a circumference equal to $\lambda$. The rough calculation confirms that an Archimedean antenna designed for the microwave band $1-20 \mathrm{GHz}$ at the working frequency of $10 \mathrm{GHz}$ has less than $0.5 \%$ aperture utilization or efficiency of radiation [22].

\section{Terahertz-Design FZP Lens Antenna: Electromagnetic and Structural Evaluation}

Next are described terahertz FZP lens and antenna, designed at the terahertz frequency of $1350 \mathrm{GHz}$ (Figure 7), or at the 15th harmonic of the $90 \mathrm{GHz}$ microwave FZP lens. Both, terahertz-design and microwave-design lenses are equal in size, or they have $D=100 \mathrm{~mm}, F=66.7 \mathrm{~mm}$, and lens aspect ratio $F / D=0.67$. The $1350 \mathrm{GHz}$ terahertz FZP lens encloses 150 Fresnel zones totally or 15 times much more than the $90 \mathrm{GHz}$ microwave FZP lens. Thus, their zone number scale ratio is $15: 1$ and is equal to the design frequency ratio $1350: 90 \mathrm{GHz}$. The outmost zone of the terahertz FZP lens has a width $\Delta b=185 \mu \mathrm{m}$.

The computed copolar and cross-polar radiation patterns of terahertz-design FZPA-T-150 versus angle $\theta$ are drawn in Figure 8, red line and blue line, respectively. The main 
TABLE 3: Radiation pattern parameters of FZP lens antennas: microwave (M) and terahertz (T) designs.

\begin{tabular}{|c|c|c|c|c|c|c|}
\hline $\begin{array}{l}\text { FZP antenna } \\
F / D=0.67\end{array}$ & $\begin{array}{c}f \\
(\mathrm{GHz}) \\
\end{array}$ & $\begin{array}{c}B W \\
(\mathrm{deg})\end{array}$ & $\begin{array}{c}\delta_{0} \\
(\mathrm{deg})\end{array}$ & $\begin{array}{c}r_{0} \\
(\mu \mathrm{m})\end{array}$ & $\begin{array}{c}L_{\mathrm{s}} \\
(\mathrm{dB}) \\
\end{array}$ & $\begin{array}{r}L_{\mathrm{cr}} \\
(\mathrm{dB}) \\
\end{array}$ \\
\hline FZPA-M-10 & 90 & 2.4 & 2.43 & 2835 & \multirow{3}{*}{11} & \multirow{3}{*}{-25} \\
\hline$\left(f_{1}=90 \mathrm{GHz}\right.$ & 270 & 0.78 & 0.81 & 945 & & \\
\hline$D=100 \mathrm{~mm})$ & 1350 & 0.15 & 0.16 & 189 & & \\
\hline \multicolumn{7}{|l|}{ FZPA-T-150 } \\
\hline $\begin{array}{l}\left(f_{1}=1350 \mathrm{GHz}\right. \\
D=100 \mathrm{~mm})\end{array}$ & 1350 & 0.15 & 0.155 & 226 & 22 & -56 \\
\hline
\end{tabular}

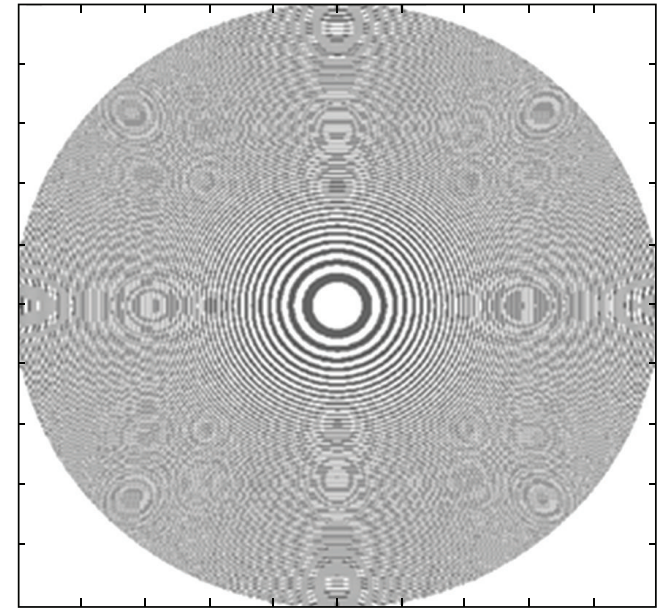

Figure 7: Terahertz-design FZP lens of FZPA-T-150 lens antenna designed at $1350 \mathrm{GHz}$.

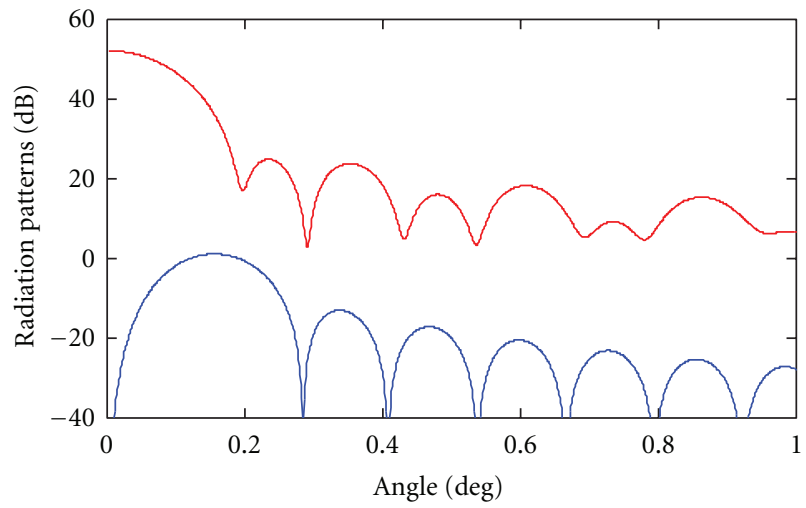

Figure 8: Radiation patterns of terahertz-design FZPA-T-150 versus angle $\theta$ : copolar radiation pattern (red line) and cross-polar radiation pattern (blue line).

frequency and radiation-pattern parameters of the $1350 \mathrm{GHz}$ antenna FZPA-T-150 are also listed in Tables 1 and 2, below the analogous parameters of the $90 \mathrm{GHz}$ microwave antenna FZPA-M-10.

At the same working terahertz frequency of $1350 \mathrm{GHz}$, both antennas, the microwave antenna FZPA-M-10 and terahertz antenna FZPA-T-150, have the following radiation parameters:

(a) similar absolute and relative bandwidths of about $16.5 \mathrm{GHz}$ (absolute) and 1.2\% (relative), respectively;

(b) comparable radiation pattern beamwidths (or angular resolutions) of about 0.15 degrees;

Naturally, the terahertz-design antenna FZPA-T-150 has a much higher gain and aperture efficiency, but a similar radiation to those of FZPA-M-10 at its design frequency of $90 \mathrm{GHz}$. At FZPA-T-150 harmonic frequencies like $4.05 \mathrm{THz}$ (3rd terahertz harmonic) and $20.25 \mathrm{THz}$ (15th harmonic, located in the low-infrared band), the aperture utilization efficiency of the FZPA-T-150 will again become low, about $1 \%$ and $0.05 \%$, correspondingly.

With regards to the structural, technological, and other lens qualities, the contrast between the microwave- and terahertz-design FZP lenses shows:

(i) FZP lens used in the FZPA-T-150 antenna has very narrow ending zones. As was pointed out above, the last or the 150th zone is only $185 \mu \mathrm{m}$ wide. For comparison, the last zone of the microwave-design FZP lens in FZPA-M-10 is $2830 \mu \mathrm{m}$ width or about 15 times broader;

(ii) microwave-design FZP lens in the FZPA-M-10 antenna working at high-terahertz harmonics has a much simpler construction and can be easily fabricated, while the terahertz-design FZP lens needs sophisticated, precise, and costly microtechnology for its production;

(iii) narrow-zone terahertz-design FZP lenses are more fragile electrically and might not withstand highenergy illumination $[18,19]$.

It is fascinating that the big microwave-design antenna FZPA-M-10 could operate at frequency harmonics much higher than the terahertz frequencies or in the infrared band, for instance, for which the antenna aperture efficiency will be really extremely low (at about $10^{-4} \div 10^{-5} \%$ ). By all criteria and applications, such efficiency values will not be pragmatic.

Instead, the terahertz lens/antenna FZPA-T-150 could be chosen for reaching the low infrared range. As is pointed out above, its 15th harmonic is the infrared frequency of $20250 \mathrm{GHz}$ (or $20.25 \mathrm{THz}$ ) that corresponds to wavelength 
of $14.81 \mu \mathrm{m}$. For a normal operation at this infrared wavelength, the thickness of the $1350 \mathrm{GHz}$ FZP has to be very thin, less than about $0.1-0.15$ wavelengths, or less than 1.5$2.0 \mu \mathrm{m}$. The linear transverse resolution $r_{0}$ of such terahertz lens/antenna operating at the infrared frequency will be around $12 \mu \mathrm{m}$.

\section{Conclusion}

The study exposes for the first time a number of intriguing features related to the binary microwave FZP lens and antenna operating at harmonic terahertz frequencies. The harmonic gain versus frequency pattern shows a strict appearance of the peak gains in proportion to the frequency harmonic sequence $90: 270: 450, \ldots, 1530 \mathrm{GHz}$. This antenna property makes the FZP lens antenna a frequency-domain filtering device. For a given microwave FZP design all harmonic gain peaks have roughly identical shapes, bandwidths, and top values. If the lens/antenna resolution is of major importance and the aperture efficiency is a secondary problem, or might be improved if necessary by additional amplification, the microwave-design FZP lens antenna can be of big importance. Unique and expensive microtechnology is not required for fabrication of simple and large in size microwave-design FZP lenses running at high-terahertz frequencies. Similarly, the terahertz-design FZP lens designs can be used for focusing in the infrared or higher optical bands. Also, the narrow-zone terahertz FZP lens designs are more fragile electrically if illuminated by high-energy fields.

The high-order harmonic operation of the FZP lens and antenna could find space resolution and frequency filtering applications in terahertz and optical metrology, imaging tomography, spectral analysis, short-range communications, synchrotron radiation and focusing facilities, and radio astronomy, among others.

\section{Acknowledgment}

The author acknowledges the Support received from the Chilean Science Agency CONICYT within the Fondecyt Project 1095012/2009 and Anillos ACT-53/2010 Project.

\section{References}

[1] I. Hosako, N. Sekine, M. Patrashin et al., "At the dawn of a new era in terahertz technology," Proceedings of the IEEE, vol. 95, no. 8, pp. 1611-1623, 2007.

[2] R. Piesiewicz, T. Kleine-Ostmann, N. Krumbholz et al., "Short-range ultra-broadband terahertz communications: concepts and perspectives," IEEE Antennas and Propagation Magazine, vol. 49, no. 6, pp. 24-39, 2007.

[3] Y.-S. Lee, Principles of Terahertz Science and Technology, Springer, Berlin, Germany, 2008.

[4] F. Sobel, F. Wentworth, and J. Wiltse, "Quasi-optical surface waveguide and other components for the 100 - to $300 \mathrm{Gc}$ region," IRE Transactions on Microwave Theory and Techniques, vol. 9, pp. 512-518, 1961.

[5] L. Leyten and M. H. A. J. Herben, "Vectorial far-field analysis of the fresnel-zone plate antenna: a comparison with the parabolic reflector antenna," Microwave and Optical Technology Letters, vol. 5, no. 2, pp. 49-56, 1992.

[6] P. Smith, Quasioptical Systems, Wiley, New York, NY, USA, 1998.

[7] M. A. Gouker and G. S. Smith, "A millimeter-wave integratedcircuit antenna based on the Fresnel zone plate," IEEE Transactions on Microwave Theory and Techniques, vol. 40, no. 5, pp. 968-977, 1992.

[8] H. D. Hristov and M. H. A. J. Herben, "Millimeter-wave Fresnel-zone plate lens and antenna," IEEE Transactions on Microwave Theory and Techniques, vol. 43, no. 12, pp. 2779-2785, 1995.

[9] H. D. Hristov, Fresnel Zones in Wireless Links, Zone Plate Lenses and Antennas, Artech House, 2000.

[10] J. C. Wiltse, "Zone plate designs for terahertz frequencies," in Terahertz for Military and Security Applications III, Proceeding of SPIE, pp. 167-179, March 2005.

[11] I. Minin and O. Minin, Diffractional Optics of Millimeter Waves, Institute of Physics, Bristol, UK, 2004.

[12] J. C. Wiltse, "Diffraction optics for terahertz waves," in Terahertz for Military and Security Applications II, Proceeding of SPIE, pp. 127-135, April 2004.

[13] J. C. Wiltse, "Dual-band Fresnel zone plate antennas," in Targets and Backgrounds: Characterization and Representation III, Proceeding of SPIE, pp. 181-185, April 1997.

[14] S. Wang, T. Yuan, E. D. Walsby et al., "Characterization of Tray binary lenses," Optics Letters, vol. 27, no. 13, pp. 1183$1185,2002$.

[15] X. C. Zhang, Q. Hu, H. Roskos, and P. Haring Bolívar, "Threedimensional terahertz wave imaging," Philosophical Transactions of the Royal Society A, vol. 362, no. 1815, pp. 283-299, 2004.

[16] I. V. Minin and O. V. Minin, "THz quasioptics applications in security," in Terahertz for Military and Security Applications $I V$, Proceedings of SPIE, April 2006.

[17] E. Schonbrun, W. N. Ye, and K. B. Crozier, "Scanning microscopy using a short-focal-length Fresnel zone plate," Optics Letters, vol. 34, no. 14, pp. 2228-2230, 2009.

[18] CERN Courier Note, "Fresnel plate sharpens focus of helium beam," March 2000.

[19] Xradia Application Note, "Fresnel zone plate lenses," http:// xradia.com/.

[20] L. C. J. Baggen, C. J. J. Jeronimus, and M. H. A. J. Herben, "Scan performance of the Fresnel-zone plate antenna: a comparison with the parabolic reflector antenna," Microwave and Optical Technology Letters, vol. 6, no. 13, pp. 769-774, 1993.

[21] O. Meyers Jr., "Studies of transmission zone plates," American Journal of Physics, vol. 19, no. 6, pp. 359-365, 1951.

[22] J. D. Kraus and R. J. Marethka, Antennas for All Applications, McGraw-Hill, 2002.

[23] D. J. Stigliani Jr., R. Mittra, and R. G. Semonin, "Resolving power of a zone plate," Journal of the Optical Society of America, vol. 57, no. 5, pp. 610-613, 1967.

[24] P. D. Kearney and A. G. Klein, "Resolving power of zone plates," Journal of Modern Physics, vol. 36, no. 3, pp. 361-367, 1989. 

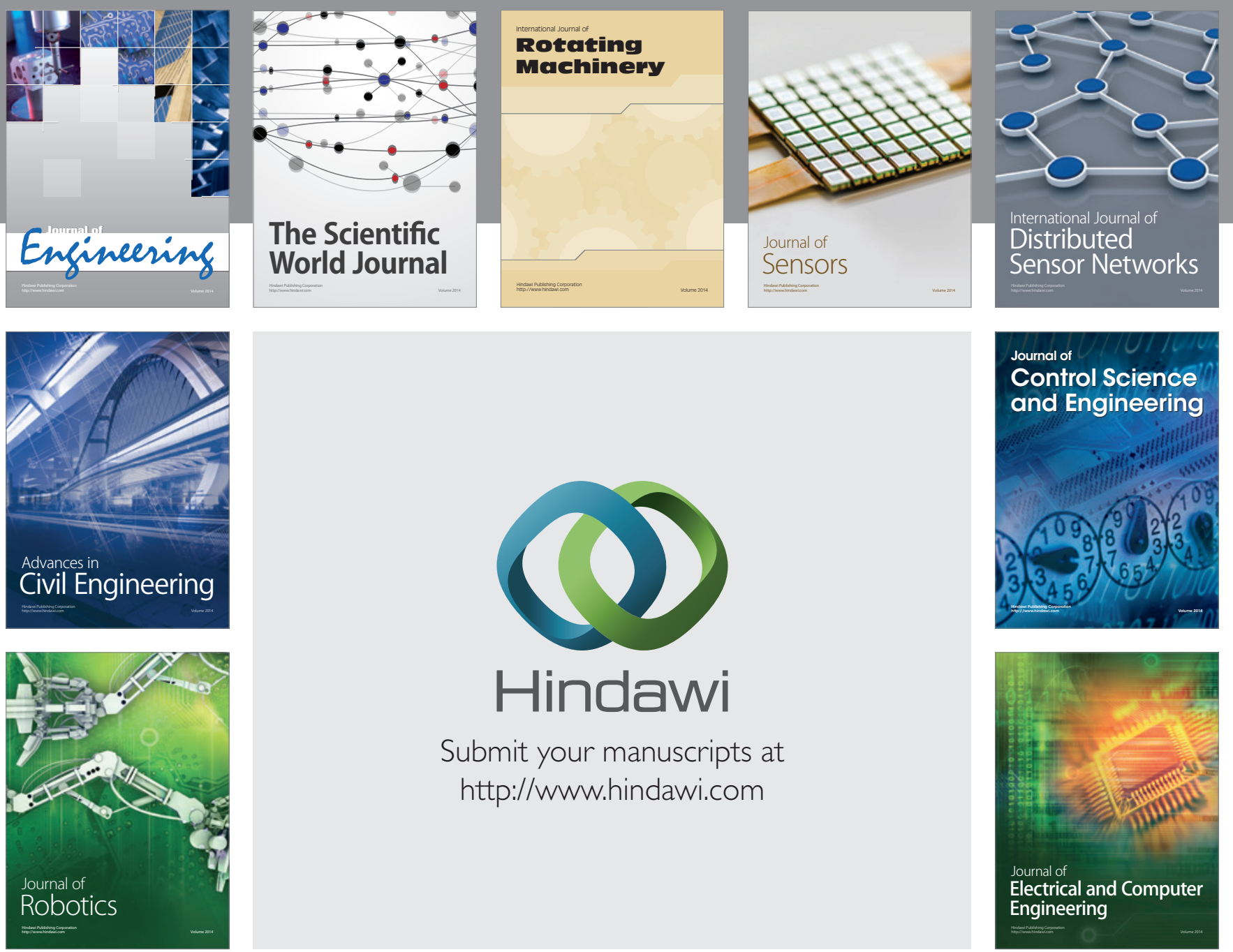

Submit your manuscripts at

http://www.hindawi.com
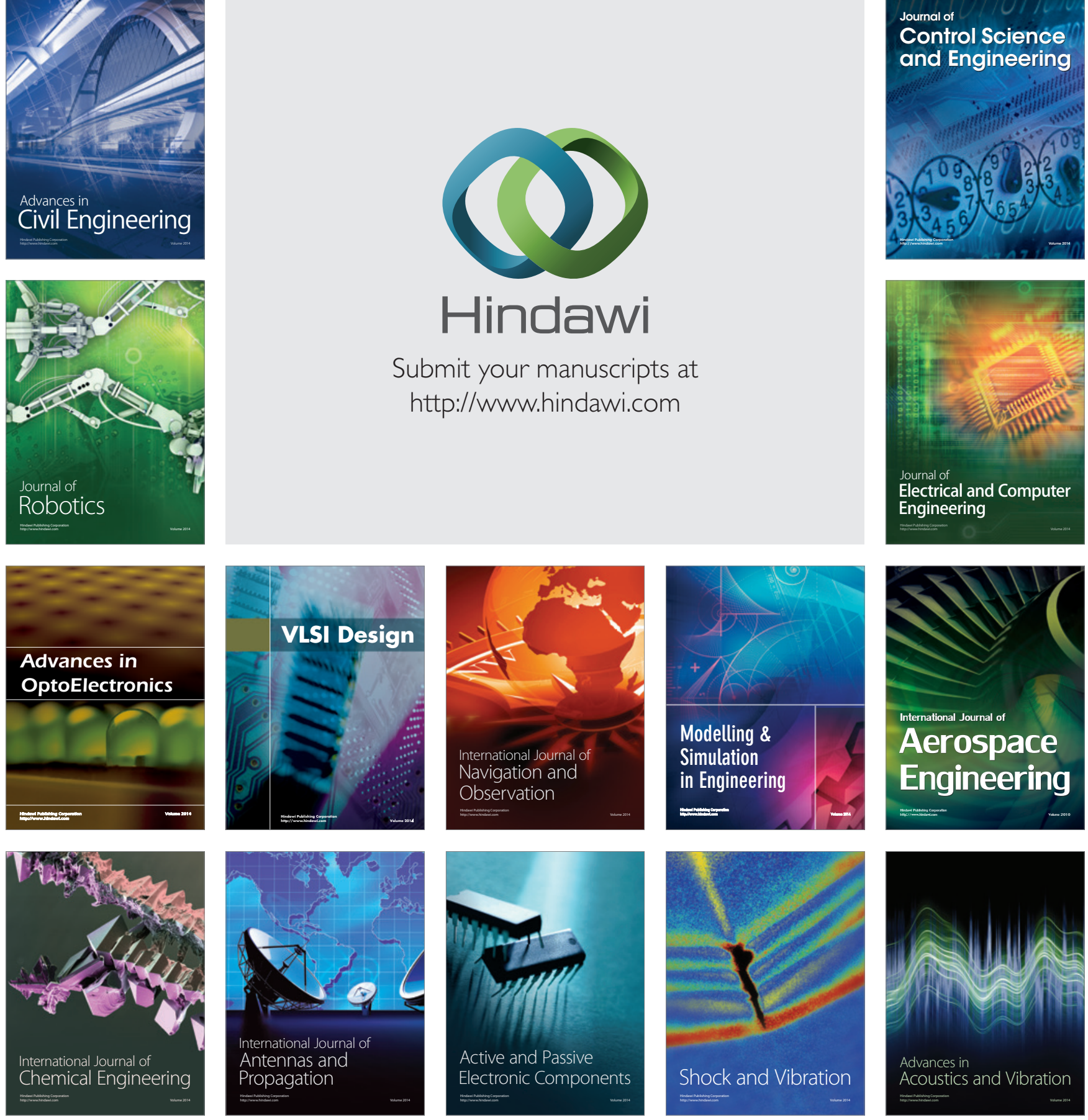